\title{
Mathematical modeling of mass transfer in critical regimes of vertical two-phase flows
}

\author{
Eugene Barsky \\ Department of Industrial Engineering \\ Azrieli College of Engineering, Israel \\ eugene@jce.ac.il
}

\begin{abstract}
A system of differential equations describing main regularities of mass transfer process in critical regimes of two-phase flows is formed. Using this system, several similarity criteria are determined. Equations of discrete-stationary solid phase distribution in such flows along the channel height are derived. The analysis of this system allowed us to define optimal conditions of the process of bulk materials classification in vertical channels.
\end{abstract}

Keywords: separation, two phase flow, narrow size class, balance model

\section{Introduction}

We call two-phase flow regimes critical, if a certain part of solid phase moves upwards with the flow, and another part settles down against the flow. This process is most widely used in industry for bulk materials fractionating according to particles sizes or densities.

Longstanding study of fractionating processes has revealed a number of interesting empirical regularities [1]. It is established that the extraction of particles of narrow size classes is invariant with respect to the initial granulometric composition of the material. It means that each size class is separated in the flow independently of other size classes. This regularity is valid at the concentrations of solid matter in the air flow up to $3 \mathrm{~kg} / \mathrm{m}^{3}$ and in water - up to $3 \mathrm{~kg} / \mathrm{liter}$.

However, here particles of various sizes intensely interact with each other and with the channel walls $[1,2,4]$.

Present-day gravitational separation apparatuses make it possible to reach sufficiently high separation efficiency by the boundary up to 10 micron.

Let us examine the relation between the concentration and the number of particles in real conditions for a narrow class, for example, with the average particle size equal to 30 micron.

Let the particles density be $\rho=2600 \mathrm{~kg} / \mathrm{m}^{3}$. If the consumed concentration $\mu=2 \mathrm{~kg} / \mathrm{m}^{3}$, volume concentration of solids in the air flow is insignificant and amounts to $7.7 \cdot 10^{-4}\left(\beta=\frac{2}{2600}\right)$ only.

One 30-micron particle weighs $0.3674 \cdot 10^{-11} \mathrm{~kg}$. We assume that the percentage of such particles in the initial composition is $10 \%$; then their content in one cubic meter is:

$$
n=\frac{0.2}{0.3674 \cdot 10^{-11}}=5,4 \cdot 10^{9} .
$$

It means that one cubic meter of air contains 5,4 milliards of such particles, which is comparable with the number of molecules in a rarefied gas. To solve many problems related to gaseous systems, the notion of an infinitesimal volume is used. Therefore, we use a similar approach in this case [3] on the basis of some well-grounded assumptions.

\section{Balance Model}

A schematic diagram of a gravitational separator is presented in Fig. 1. The initial material is fed to a vertical channel. A moving medium flow moves upwards in the channel at the average velocity $\mathrm{w}$. The fine product is transferred upwards, and the coarse one settles down, with particles of certain size classes being divided in various proportions between these products. 
Clearly, the finest classes are practically totally carried upward by the flow, while the coarsest classed are almost totally settled down. Everything depends of the ratio between the flow velocity and particle sizes [1].

Classes of medium-size particles are divided in a smaller proportion. We can even imagine (which is quite realistic) such class of particles which is divided half-and-half between both outlets.

We consider an infinitesimal contour, which is immobile with respect to channel walls (Fig. 2). It represents a parallelepiped with the edges $d x ; d y ; d z$.

Let us consider a flow of particles of a certain narrow size class $j$ per an infinitesimal period of time $d t$ along the axis $z$. At the level of the lower surface $d y d x$, the content of particles of this narrow size class in a unit volume is $n$ (for convenience, we omit the index $j$ in further derivations).

During the time period $d t, n v_{z} d x d y \cdot d t$ particles enter the contour through this face, where $v_{z}$ is the velocity of particles of the given size along $z$ axis. The number of particles leaving the contour through the upper face is

$$
\left(n v_{z}+\frac{\partial\left(n v_{z}\right)}{\partial z} d z\right) d x d y d t
$$

The difference between these two flows is

$$
-\frac{\partial\left(n v_{z}\right)}{\partial z} d x d y d z d t=-\frac{\partial\left(n v_{z}\right)}{\partial z} d V d t
$$

where $d V=d x d y d z$ is the volume of the contour.

We can get similar differences for other axes, too, in the form

$$
-\frac{\partial\left(n v_{z}\right)}{\partial x} d V d t \quad \text { and } \quad-\frac{\partial\left(n v_{y}\right)}{\partial x} d V d t
$$

Let us find the total change in the number of particles within the contour during the time period $d t$. We assume that at a certain initial moment of time $t$, the number of particles within the contour amounts to $n d V$. Since $n$ can vary both with coordinates $(x ; y ; z)$ and with time $(t)$, the change in this number by the moment $t+d t$ amounts, in partial derivatives, to

$$
\frac{\partial(n d V)}{\partial t} d t=\frac{\partial n}{\partial t} d V d t
$$

Now we collect all balance elements within the same equation reducing by $d V d t$. As a result, we obtain

or

$$
-\frac{\partial n}{\partial t}=\frac{\partial\left(n v_{x}\right)}{\partial x}+\frac{\partial\left(n v_{y}\right)}{\partial y}+\frac{\partial\left(n v_{z}\right)}{\partial z}
$$

$$
\frac{\partial n}{\partial t}+\frac{\partial\left(n v_{x}\right)}{\partial x}+\frac{\partial\left(n v_{y}\right)}{\partial y}+\frac{\partial\left(n v_{z}\right)}{\partial z}=0
$$

Clearly, the integration of (1) with specified initial conditions (single-valuedness condition) leads to the conservation law. 


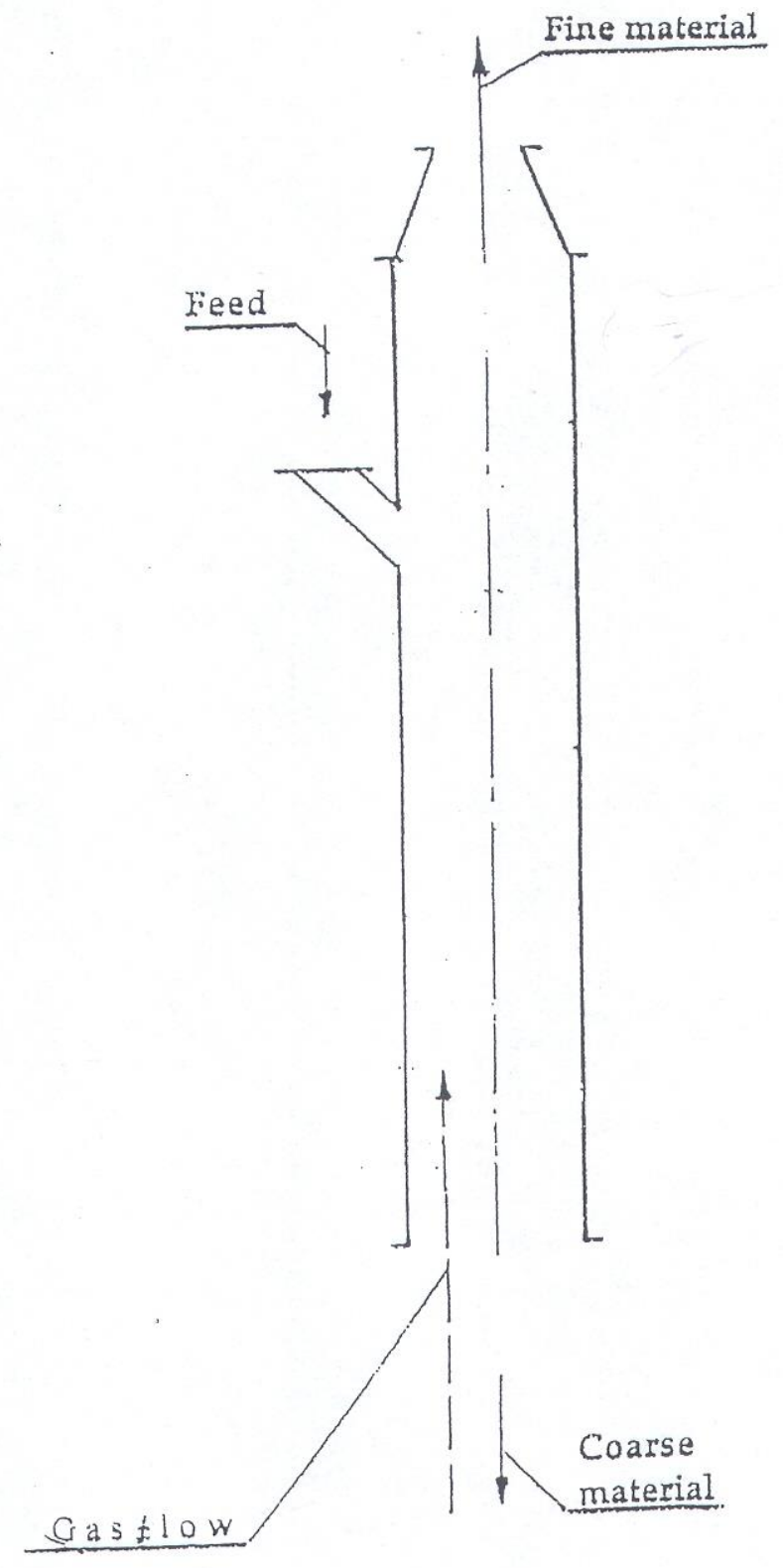

Fig. 1. Schematic diagram of gravitational separator. 


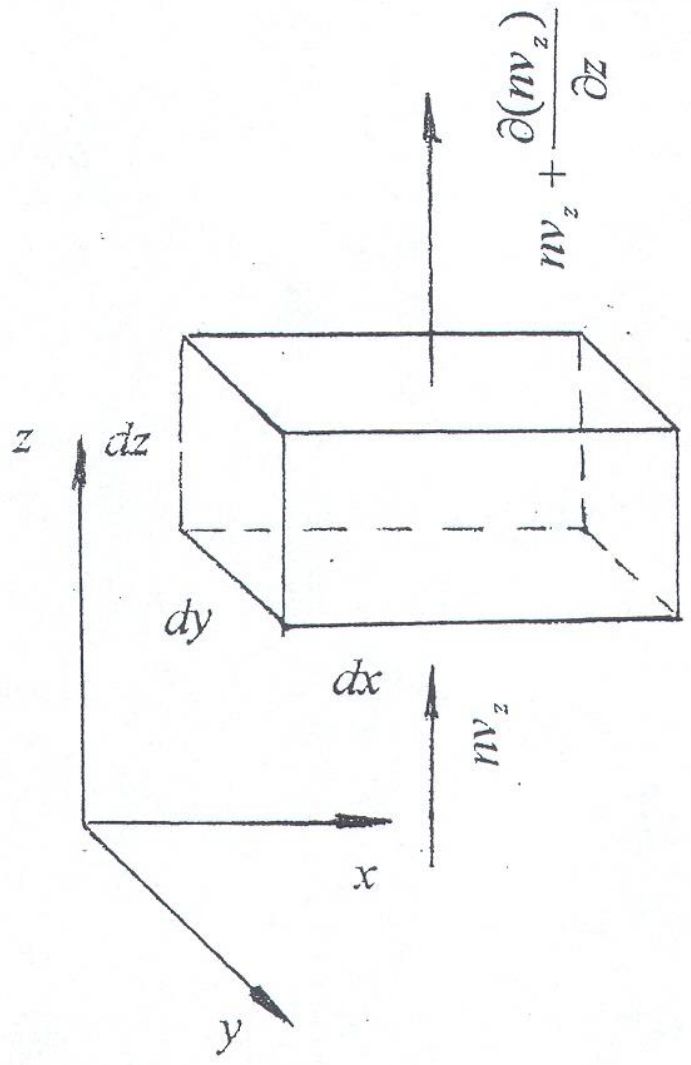

Fig. 2. Material flow through the contour. 


\section{Transverse transfer}

Besides the longitudinal particles transfer, a transverse migration of particles takes place in a two-phase flow. It has been studied since long ago and reduced to the phenomenon of diffusion [4]. This assumption is rather relative, since it is caused not only by the gradient of particles concentration in various points inside the flow.

This phenomenon is also affected by the velocity gradient, collisions of particles, turbulence and other causes.

We examine a vertical axis $z$ in the flow and a motionless contour (Fig. 2).

We assume that the particles concentration at the left face is $n$, and at the right face $-n+\frac{\partial n}{\partial x} d x$. Then, due to said reasons, particles transfer takes place. Their arrival to the volume $d v$ during the time $d t$ through the left face is expressed as follows:

$$
-D \frac{\partial n}{\partial x} d x d y d z
$$

where $D$ is the proportionality factor (diffusion coefficient).

Their exit through the right face is

$$
-D \frac{\partial}{\partial x}\left(n+\frac{\partial n}{\partial x} d x\right) d y d z d t
$$

The difference between these flows amounts to

$$
D \frac{\partial^{2} n}{\partial x^{2}} d x d y d z d t=D \frac{\partial^{2} n}{\partial x} d V d t
$$

Similar flows along other axes $y$ and $z$ are also possible, and the differences along these axes are

$$
D \frac{\partial^{2} n}{\partial y^{2}} d V d t \text { and } D \frac{\partial^{2} n}{\partial z^{2}} d V d t \text {. }
$$

The total difference in the amounts of particles at the contour inlet and outlet in three directions amounts to

$$
D\left(\frac{\partial^{2} n}{\partial x^{2}}+\frac{\partial^{2} n}{\partial y^{2}}+\frac{\partial^{2} n}{\partial z^{2}}\right) d V d t=D \nabla^{2} n d V d t
$$

On the other hand, as a result of the transverse transfer, in the contour with the volume $d V$ the particles concentration is changed by $d n d V$.

Now we bring all balance elements together into one equation and, after reducing by $d V d t$, obtain

$$
\frac{\partial n}{\partial t}=D \nabla^{2} n
$$

Since $n$ changes with coordinates and time, we can write:

$$
d n=\frac{\partial n}{\partial t} d t+\frac{\partial n}{\partial x} d x+\frac{\partial n}{\partial y} d y+\frac{\partial n}{\partial z} d z
$$

We divide both parts of the latter equality by $d t$, make the following substitutions

and finally obtain:

$$
\frac{d x}{d t}=v_{x} ; \quad \frac{d y}{d t}=v_{y} ; \quad \frac{d z}{d t}=v_{z}
$$

$$
\frac{\partial n}{\partial t}=v_{x} \frac{\partial n}{\partial x}+v_{y} \frac{\partial n}{\partial y}+v_{z} \frac{\partial n}{\partial z}=D \nabla^{2} n
$$




\section{Similarity criteria for mass transfer process}

For a direct solution of equations (1) and (2) in the general form, actual data are lacking. Therefore, we try to do it using similarity criteria for the process under study.

According to classical definitions, two physical phenomena are similar, if analogous physical characteristics in similar points of geometrically similar systems differ only by constant factors (similarity factors) [5]. The mathematical description of such systems should be identical.

First, we consider the balance equation (1).

For two similar points of similar systems, we can write:

$$
\begin{aligned}
& \frac{\partial n_{1}}{\partial t_{1}}+\frac{\partial\left(n_{1} v_{z_{1}}\right)}{\partial z_{1}}+\cdots=0 \\
& \frac{\partial n_{2}}{\partial t_{2}}+\frac{\partial\left(n_{2} z_{2}\right)}{\partial z_{2}}+\cdots=0
\end{aligned}
$$

Two phenomena are similar, if the following equalities are valid for each pair of similar points:

$$
\begin{aligned}
& \frac{n_{1}}{n_{2}}=m_{n} ; \quad \frac{t_{1}}{t_{2}}=m_{t} ; \quad \frac{v_{z_{1}}}{v_{z_{2}}}=m_{v} ; \quad \frac{z_{1}}{z_{2}}=m_{e}, \quad \text { i.e. } \\
n_{1}= & m_{n} n_{2} ; \quad t_{1}=m_{t} t_{2} ; \quad v_{z_{1}}=v_{n_{v}} v_{z_{2}} ; \quad z_{1}=z_{2} m_{e}
\end{aligned}
$$

Each similarity factor serves as a scaling characteristic of the respective physical magnitude. The similarity factor denoted by $m_{e}$ is common for all coordinates.

Equation (3) can be written in the form:

$$
\frac{m_{n}}{m_{t}} \frac{\partial n_{2}}{\partial t_{2}}+\frac{m_{n} m_{t}}{m_{e}} \frac{\partial\left(n_{2} v_{z_{2}}\right)}{\partial z_{2}}+\cdots=0
$$

The latter equation (6) describes the system (3) having characteristics of the system (4).

Similar systems should be described by the same equations. It is possible only if the similarity factors in equation (6) can be factored out (or reduced). It means that they should be equal, i.e.

Clearly, it can be reduced by $m_{n}$ and obtain

$$
\frac{m_{n}}{m_{t}}=\frac{m_{n} m_{t}}{m_{e}}
$$

$$
\frac{1}{m_{t}}=\frac{m_{t}}{m_{e}}
$$

Now we substitute the values of similarity criteria from (5):

$$
\frac{t_{2}}{t_{1}}=\frac{v_{z_{1}} z_{2}}{v_{z_{2}} z_{1}}
$$

and bring together magnitudes with the same indices:

$$
\frac{v_{z_{1}} t_{1}}{z_{1}}=\frac{v_{z_{2}} t_{2}}{z_{2}}=\frac{v_{z} t}{z}=\text { idem }
$$

The last ratio in (7) has no indices, which implies the validity of this equality for all similar flows. Note that this ratio is dimensionless; it is known as homochronism criterion and denoted by the symbol

$$
H_{0}=\frac{v_{z} t}{z}
$$


This criterion reflects the ratio between competing effects in the process under study,-i.e. particles accumulation and transfer in the target volume. The criterion (8) is an important physical parameter allowing us to establish the process relaxation time and the transition to a stationary mode. It stops operating in stationary modes.

Let us examine Equation (2) from the same standpoint. It is sufficient to represent it in the following form:

$$
\frac{\partial n_{1}}{\partial t_{1}}+\left(v_{z_{1}} \frac{\partial n_{1}}{\partial z_{1}}+\cdots\right)=D_{1}\left(\frac{\partial^{2} n_{1}}{\partial z_{1}^{2}}+\cdots\right)
$$

Let us write equalities similar to (5) for thus equation:

$$
n_{1}=m_{n} n_{2} ; \quad t_{1}=m_{t} t_{2} ; \quad v_{z_{1}}=m_{v} v_{z_{2}} ; \quad z_{1}=m_{e} z_{2} ; \quad D_{1}=m_{D} D_{2}
$$

We can write this equation using symbols of the similar system as follows

$$
\frac{m_{n}}{m_{t}} \frac{\partial n_{2}}{\partial t_{2}}+\left(\frac{m_{v} m_{n}}{m_{e}} v_{z_{2}} \frac{\partial n_{2}}{\partial z_{2}}+\cdots\right)=\frac{m_{D} m_{n}^{2}}{m_{e}^{2}} D_{2}\left(\frac{\partial^{2} n_{2}}{\partial z_{2}^{2}}+\cdots\right)
$$

Here the similarity factors should be also equal, so that we could factor them out, i.e.

We can obtain three similarity criteria:

$$
\frac{m_{n}}{m_{t}}=\frac{m_{v} m_{n}}{m_{e}}=\frac{m_{D} m_{n}^{2}}{m_{e}^{2}}
$$

$$
\frac{1}{m_{t}}=\frac{m_{v}}{m_{e}}
$$

whence

$$
\frac{t_{2}}{t_{1}}=\frac{v_{z_{1}} z_{2}}{v_{z_{2}} z_{1}}=\frac{v_{z} t}{z}
$$

This is the same homochronism criterion:

$$
\frac{1}{m_{t}}=m_{D} \frac{m_{n}}{m_{e}^{2}}
$$

whence

$$
\frac{t_{2}}{t_{1}}=\frac{D_{1}}{D_{2}} \frac{n_{1} z_{2}^{2}}{n_{2} z_{1}^{2}}
$$

We can write:

$$
n_{1} \frac{D_{1} t_{1}}{z_{1}^{2}}=n_{2} \frac{D_{2} t_{2}}{z_{2}^{2}}=n \frac{D t}{z}=\text { idem }
$$

This criterion can be also used for describing non-stationary hydrodynamic mass transfer processes:

$$
\frac{m_{v} m_{n}}{m_{e}}=m_{D} \frac{m_{n}^{2}}{m_{e}^{2}}
$$

whence

$$
m_{v}=\frac{m_{D} m_{n}}{m_{e}}
$$

Basing on this, we can write: 


$$
\frac{v_{z_{1}}}{v_{z_{2}}}=\frac{D_{1} n_{1} z_{2}}{D_{2} n_{2} z_{1}}
$$

whence

$$
n_{1} \frac{D_{1}}{v_{z_{1}} z_{1}}=n_{2} \frac{D_{2}}{v_{z_{2}} z_{2}}=n \frac{D}{v_{z} z}=\text { idem }
$$

This criterion shows the relation between the amounts of diffusion-transferred matter and velocity fluxes.

By combining these parameters, other criterial relations can be derived. For example, multiplying expressions (8) and (10), we obtain:

$$
\frac{v_{z} t}{z} \cdot n \frac{D}{v_{z} z}=n \frac{D t}{z^{2}}=i d e m
$$

This dependence is called Zhukovsky's criterion $(Z h)$.

Thus, the solution of the mathematical mass transfer model in the critical regime of two-phase flows has given us a number of generalized parameters for the description of such flows.

However, the importance of the derived differential equations (1) and (2) extends further, - e.g., their direct solution is of certain interest. And although it is quite clear that it is impossible to get their general solution due to the lack of necessary data, their partial solutions can also provide important information.

\section{Partial solution of principal differential equations}

Under certain conditions, Equations (1) and (2) can be significantly simplified. First, a stationary process, where all characteristics of a two-phase flow are independent of time, is of utmost interest. Under such conditions,

$$
\frac{\partial n}{\partial t}=0
$$

Secondly, we can restrict ourselves with a quasi-one-dimensional model of transfer along $z$ axis, because it defines the result of separation.

No reliable experimental data are available as yet for establishing the character of transfer along transverse axes $(x, y)$. Therefore, in this solution we assume that particles concentration varies only with height, and remains the same over the cross-section at any level; besides, they are uniformly distributed over this cross-section.

In this case, for (1)

$$
\frac{\partial\left(n v_{x}\right)}{\partial x}=\frac{\partial\left(n v_{y}\right)}{\partial y}=0
$$

while for (2) this condition is written as follows:

$$
\frac{\partial n}{\partial x}=\frac{\partial n}{\partial y}=0
$$

The following remains from Equation (1):

$$
\frac{d\left(n v_{z}\right)}{d z}=0
$$

and from Equation (2):

$$
v_{z} \frac{d n}{d z}=0
$$

Mean flow velocity in a channel with an invariable cross-section is constant; therefore, these two expressions are identical and can be written as 


$$
\frac{d n}{d z}=0
$$

This dependence reflects a certain condition of optimal distribution of solid particles in a flow with respect to the channel height.

The solution of Equation (12) allows an optimal organization of the process of gravitational separation of particles in vertical channels.

\section{Conclusions}

1. On the basis of mass transfer regularities in critical two-phase flows, several similarity criteria for this process are determined.

2. It is found that in case of one stage, $k=0.5$ corresponds to the optimality condition.

3. It is established that for an efficient separation of bulk materials, they should be initially fed into the middle portion of the channel.

4. It is demonstrated that the separation efficiency depends on the channel height. However, this dependence is limited. An excessive increase in height does not provide a proportional growth of the effect.

\section{Acknowledgements}

A short acknowledgement section can be written between the conclusion and the references. Sponsorship and financial support acknowledgments should be included here. Acknowledging the contributions of other colleagues who are not included in the authorship of this paper is also added in this section. If no acknowledgement is necessary, this section should not appear in the paper.

\section{References}

[1] Barsky E., Critical Regimes of Two Phase Flows in Polydisperse Solid Phase, Springer, 2010.

[2] Khoury F.M., Predicting the Performance of Multistage Separation Processes, CRC Press, 2000.

[3] Barsky E, Efficiency of Separation of Pourable Material, High Temperature, vol.47, no.6, pp.856-862, Springer, 2009.

[4] Seader J.D., Henley E.J., Separation Process Principles, John Wiley and Sons, 1998.

[5] Kittel C., Thermal Physics, John Wiley and Sons, 1977. 


\section{Figure captions}

Fig. 1. Schematic diagram of gravitational separator.

Fig. 2. Material flow through the contour.

Fig. 3. a-mass transfer between stages;

$b$ - separation diagram within one stage.

Fig. 4. Fractional separation $F_{f}(x)$ dependence on the distribution parameter $(k)$. 